\begin{tabular}{ccc}
\hline & International Journal of Engineering \& Technology, $7(2.24)(2018) 24-28$ \\
SPC & International Journal of Engineering \& Technology \\
\hline
\end{tabular}

\title{
A Robust Image Watermarking Method based on Contourlet Transform for Correcting Geometric Attacks
}

\author{
Abhinaya. M ${ }^{1 *}$, A. Umamakeswari ${ }^{2}$ \\ ${ }^{1}$ M.Tech., Computer Science and Engineering, ${ }^{2}$ Associate Dean-CSE \\ School of Computing, SASTRA Deemed to be University, Thanjavur-613401 \\ *Corresponding Author E-mail: abhiinayamukesh02@gmail.com
}

\begin{abstract}
A watermarking technique is proposed using Discrete Wavelet Transform (DWT), Discrete Cosine Transform (DCT), and Singular Value Decomposition (SVD) techniques to implement information hiding. Embedding can be done by dividing the host image into four rectangular segments which are non-overlapping called sub-images using hybrid scheme. Redundancy reduces cropping attack. The main aim is to reduce the effect of geometric bouts, such as rotation, translation, and affine translation using synchronization technique. Watermarks can be a binary random sequence of different lengths. Data replication and hamming code are two error modification methods utilized in the proposed scheme. Achieved results show comparable robustness against geometric attacks and signal processing. Contourlet transform can be used to improve the visual feature of the image. After embedding the image or data, contourlet transform is applied in the frequency domain to improve robustness. The optimal mapping function is obtained using contourlet transform which increases Peak Signal to Noise Ratio (PSNR) and hiding capacity with low distortion.
\end{abstract}

Keywords: Discrete Wavelet Transform (DWT), Geometric attacks, Contourlet Transform, Image Watermarking, Singular Value Decomposition (SVD).

\section{Introduction}

The best solution for authenticity and identity is Image Watermarking. Image watermarking is capable of resisting illegal usages. Secret data is hidden inside a host cover (watermarked) image. Secret data detection proposes copyright. The important characteristics of watermarking provide (1) Imperceptibility (i.e., there should be no visual compromise) and (2) Robustness against various attacks. There are two domains namely spatial or signal domains in watermarking. Altering pixels pave a way to low-cost design called spatial domain watermarking. Spatial domain is not robust to signal processing and affine transformations.

Usages of transform domain avoid signal processing attacks. Transform coefficient is modified, and the watermark is embedded, and when the inverse transform is obtained, irregularity over the image can be seen. Specifications of transform domain schemes are (1) Spatial and frequency domain are localized in DWT, (2) Extraction of geometric features is done by employing a mathematical method in case of SVD, and (3) Computation cost is low and energy compaction is high in case of DCT. The performance of watermarking is enhanced when two or more transformations are combined. By combining DWT, DCT, SVD, the robustness of watermarking approach can be obtained.

To guarantee the security, Arnold Transform is used to scramble the watermark before inserting. Additional mechanisms are provided to remove the geometric distortions. Algorithms like Fourier-Mellin Transform, Geometric Moments, Radon Transform, and Zernike Moments to reduce geometric attacks. The performance of scaling and rotation attacks is degraded by cropping attack. The focus of watermarking is mainly in either signal processing or geometric distortions. Without knowledge of the attack, when two extracted watermarks are compared, the receiver cannot identify the original watermark.

This paper illustrates the watermarking technique in the transform domain that uses contourlet transform. The method which provides robustness against signal processing and geometric attacks is used in this paper, and the main aim is to offer digital watermarking technique which has good hiding capacity and transparency. The visual perception of the image is built by representing unique identities in the name of capacity, and it captures enough information. The perception of the watermarked image should not be disturbed.

Section 2 explains the related works on contourlet transform, Methodology is given in Section 3, and Section 4 discusses the results and analysis of proposed method.

\section{Related works}

Image watermarking plays vital role in the research field for two main reasons. Firstly, it is easily available. Secondly, it carries a bulk of information where watermark can be embedded. Transform, and spatial domain differs regarding frequencies and pixels respectively. Frequency representation of the image can be done using reversible transforms like DFT, DCT, and DWT. Each has its features and represent a model differently [1].DCT technique is robust for image processing operations which are simple like low pass filtering, blurring, contrast adjustment, etc. It is more expensive and weak against cropping, rotation, scaling, etc.., DCT technique is of two types: Global and Block based. In this proposed work, a Global approach is used where embedding is done in perceptually substantial portion. The compression schemes remove a perceptually insignificant part. 
Diagonal, vertical and horizontal are three spatial directions present in the wavelet transform [2]. Filter convolution is used to implement wavelet transform, and it is computationally efficient. Embedding should be done in the lower frequency region, and decomposition level is smaller for higher frequency bands [3] Patterns and edges are located in the sub-bands have high resolution. DWT scheme is similar to DCT, the transformation process differs and results in different coefficients. Transformation of an image can be done using wavelet filters. The image is decomposed into several frequencies using filters. Decoder requirements are classified into Blind and Non-blind detection. The original image is not required for blind detection, and the original image is needed for Non-blind detection.

DFT provides robustness for geometric attacks like rotation, cropping, and scaling. The magnitude and phase portion of an image is obtained from the complex value of the real picture. Phase portion of an image gets affected by spatial shifts but not the magnitude portion. Even circular variations don't change magnitude portion. Synchronization is not required when embedding of the watermark is done in the magnitude portion. It is resistant to cropping attack. Embedding is done in a low-frequency band which is a central component of DFT [4]. Amplification is achieved through scaling of image and cyclic shift is achieved through rotation of the image. In the frequency region, rotation in spatial domain causes same effect [5].

FFT is robust against compression. Embedding algorithm is based on the template. Rather than a model, ownership is proved using an informative watermark. Even after geometric distortion, the original location is retrieved using template and extraction can be done. Implementation is complicated [6]. Multi-resolution transform proposes DHT watermarking techniques. Initially transform is applied to divide the image into different frequency bands. Embedding of the watermark is done in the lower frequency band, and the most significant component is altered to phase part Amplitude modulation is less robust to noise than phase modulation, where the watermark is embedded [7].

The Watermark detected, and the information transferred by the watermark may sometimes lead to attacks. Many classes of attacks are available [8].Watermark information is entirely removed from the watermarked data without knowledge of the watermarking process. The goal of removal attack is to impair the watermark Attacked document quality increases by keeping optimized operations like quantization. In geometric attack, instead of removing the embedded watermark, synchronized watermark information intends to distort the watermark. Perfect synchronization is used by the detector to regain the embedded watermark information. In cryptographic attack, the embedded watermark information is removed by breaking the security methods or embedded by the impair watermark. An example is brute-force attack which searches for secret information. Because of high computational complexity, application of attacks is restricted. In protocol attack, the entire logic of watermark application is attacked and it follows the concept of an invertible watermark. From the watermarked data, attacker removes his watermark and demands to be the owner of the watermark. Ambiguity occurs, because of difference in the actual ownership [9].

\section{Proposed system}

Based on image In-painting, the prediction-based reversible watermarking scheme is used to embed the secret information. Reference pixel depends on the image content distribution characteristics. Image in-painting technique uses Partial Differential Equation (PDE). Prediction image has been generated which is similar to cover image in structural and geometric information manner. Retrieval of the cover image is lossless. The in-painting algorithm requires more execution time, and it is computationally complex. Contourlet transform can be used to improve the visual feature of the image as given in Figure1a. The secret image and the input image is embedded along with the secret key. Then the obtained watermark image is used for extraction process. Contourlet transform is applied in the frequency domain to enhance robustness of the watermark image as shown in Figure1b. The difference in the error between watermarking and cover image is reduced which increases PSNR and hiding capacity with low distortion. The optimal mapping function is obtained using contourlet transform.

Contourlet transform captures the critical element of visual information named intrinsic geometrical structure. The type of digital data is discrete and finding the geometry of an image is a significant challenge. The difference between the Curvelet and the contourlet transform is that Curvelet initially starts with continuous domain and depends on the sampled data it discretizes the information but Contourlet begins with the discrete area and proceed onto the constant area. Contourlet transform uses filter bank to generate multi-direction and resolution expansion of an image with the help of contour segments. [11]

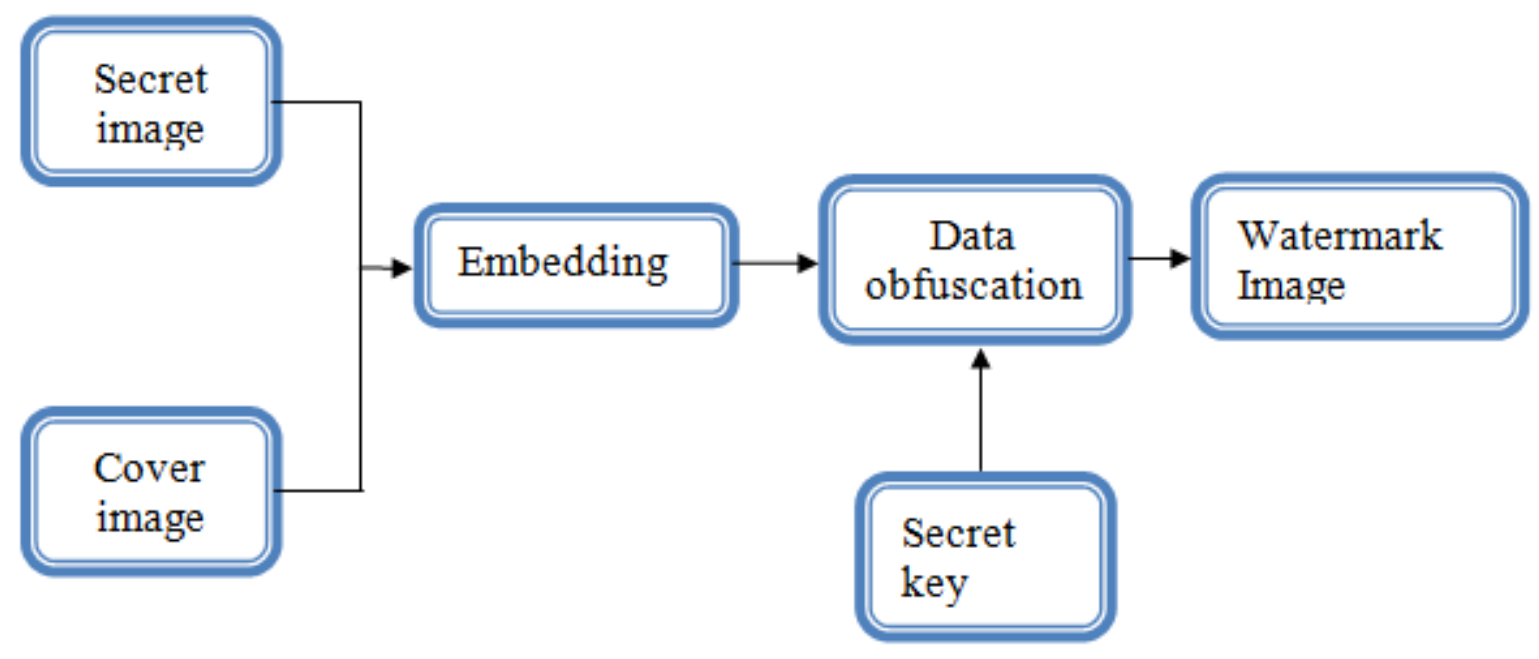

Fig. 1: (a) Embedding of Proposed system 


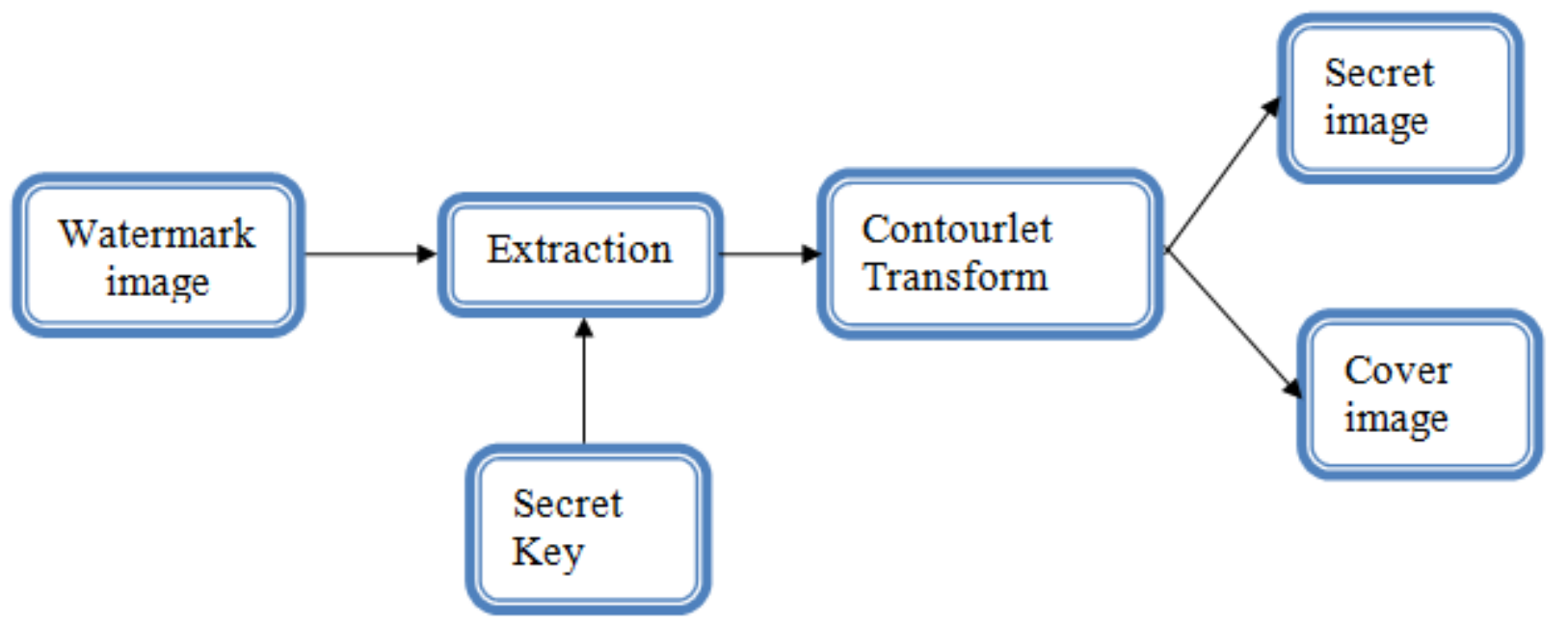

Fig. 1: (b) Extraction of Proposed system

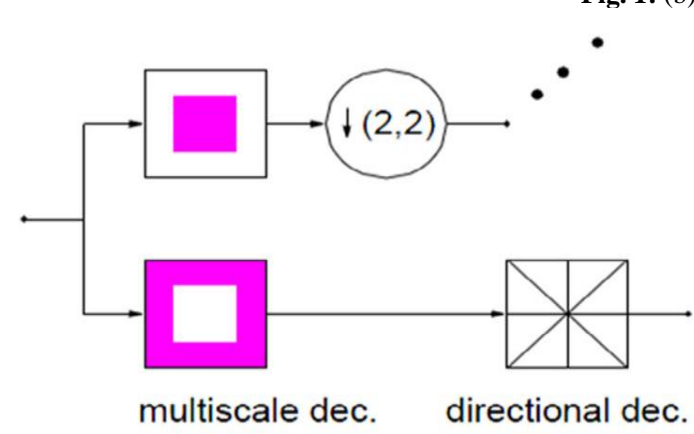

Fig. 2: (a) Block diagram of filter bank in contourlet transform

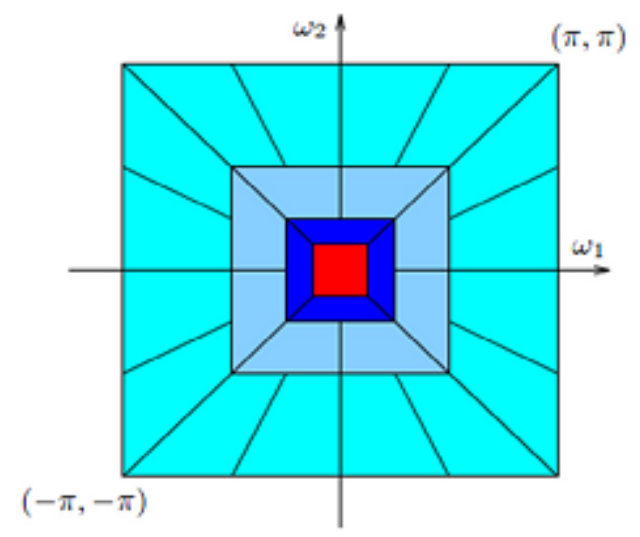

Fig. 2: (b) frequency division style

The block diagram of the filter bank and the frequency division style is shown in Figure 2 (a) and (b) respectively. Contourlet uses second filter bank to obtain smooth contour among images. Directional Filter Bank (DFB) and Laplacian Pyramid (LP) are two types of the double filter bank. To get the point discontinuities, LP is first applied followed by DFB. At each level, LP splits the image into two types, namely bandpass and low pass images. The input to the DFB is bandpass image, and directional subbands are obtained using low pass images through LP and DFB. [12]

Contourlet transform recognizes smooth contours, but wavelet fails to acknowledge it, in spite of having unique features like timefrequency and multiscale localization as in contourlet transform. Filter banks are used for efficient computation. Some structures like curvelet, ridgelet are not flexible to have multiple directions at each level. So, in discrete domain contour is introduced. [13]

\subsection{Embedding Procedure}

The image should be destroyed by Rotation, Translation and Affine Transformation and the ruined image are used for the further purpose, and MATLAB environment obtains it. The margin surrounds the central region of the image, and it consists of pixels with same intensity value. Margin invokes edges, and it acts as the boundary for the main region [20]. Then the watermarked image is embedded into four different segments of an input image, and the decomposition reduces cropping effect. Each portion of an input image is referred as sub-image.

a. The entire image is surrounded by the margin to guarantee that the required margin surrounds the main region. Edge detection operator is applied to the whole image, and canny edge detector uses a multi-stage algorithm to identify edges in images.

b. The entire image is split into four segments, and these four corners identify the minimum distance. The layers are up-left, bottom-left, up-right, bottom-right as shown in Figure 3a.

\begin{tabular}{|l|l|}
\hline LL & LH \\
\hline HL & HH \\
\hline
\end{tabular}

Fig. 3: (a) Single Level Decomposition

c. Recognition must be given to each corner of the segments and labeling can be done as shown in Figure 3c:

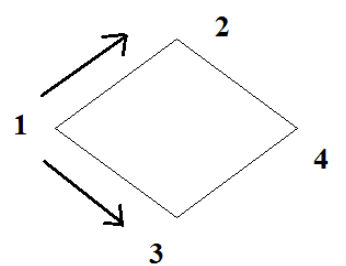

Fig. 3: (c) Labeling of corners

d. Pixels inside the region are used to reconstruct the unattacked image. The number of coordinates and their middle points of the digital images is integers. Calculation of the number of discrete points can be done using the formula:

$\mathrm{L}=\sqrt{(e 1-h 1)^{2}+(e 2-h 2)^{2}}+1$

e. To calculate the coordinate of the points:

$$
\mathrm{P}_{1}^{\mathrm{i}}=\mathrm{h} 1+\text { round }\left(\frac{(i-1)(e 1-h 1)}{L-1}\right)
$$

f. Four sub-bands: HL, LH, LL, and HH is obtained when DWT is applied on one of the sub-image using Haar filter. The size of each sub-image is $M / 4 * N / 4$. Haar filter includes low pass and high 
pass filter. The concentration of energy is high in LL band. Watermark is embedded in LH and HL bands.

g. Three sub-matrices are obtained when SVD operation is applied on matrix.

$$
\left[\begin{array}{lll}
\mathrm{U} & \mathrm{S} & \mathrm{V}
\end{array}\right]=\operatorname{SVD}(\mathrm{A})
$$

h. Matrix S is modified, and on the adjusted singular values SVD operation is again applied. Modified matrix is calculated as:

$$
\text { A_W }=U_{-} S^{\prime}{ }_{w} V^{T}
$$

i. Contourlet transform is used to obtain different sub-bands with $\mathrm{L}$ levels. Watermark bit is repeatedly embedded into different sub-bands.

j. Altered coefficients are placed in a respective position, and inverse DWT is applied to obtain watermarked sub-image.

\subsection{Extracting Procedure}

Geometric distortion procedure is used again on watermarked image. Regained watermarked image is divided into four subimages. All the steps followed in embedding phase are repeated in extraction phase except few:

a. Distorted matrix is extracted, and Inverse Arnold transform is applied. Other copies of the watermark are obtained by repeating the same procedure, and the final extracted watermark is given as:

$\mathrm{W}^{*}=(\mathrm{W} 1 *+\mathrm{W} 2 *+\mathrm{W} 3 *+\mathrm{W} 4 *) / 4$

b. Apply inverse contourlet transform to each sub-band. Repeat the steps to get all partitions of an image and watermark is extracted from the sub-band.
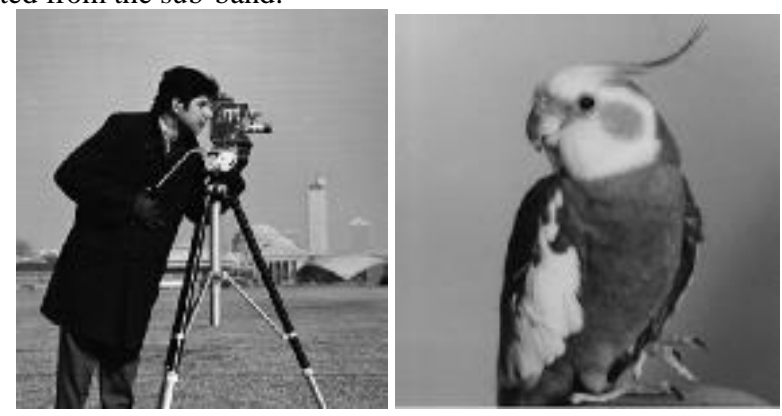

(a) Cameraman

(b) $\mathrm{Coco}$

Fig. 4: Original images

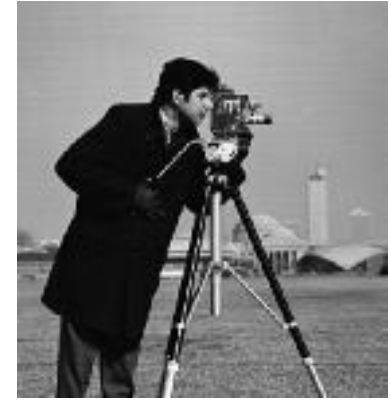

(a) Cameraman

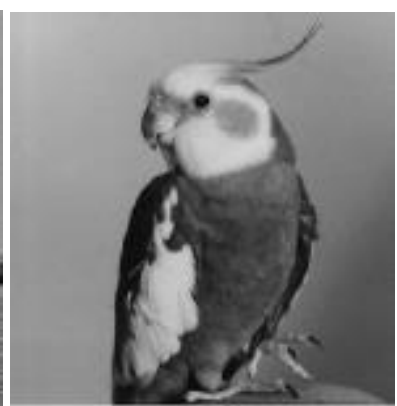

(b) $\mathrm{Coco}$

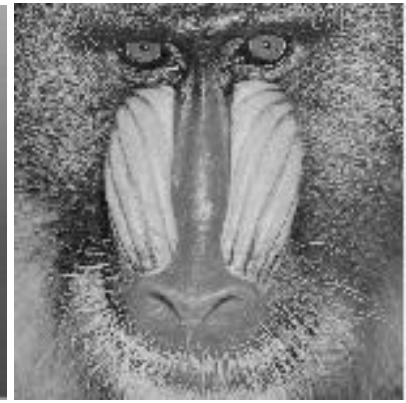

(c) Mandrill

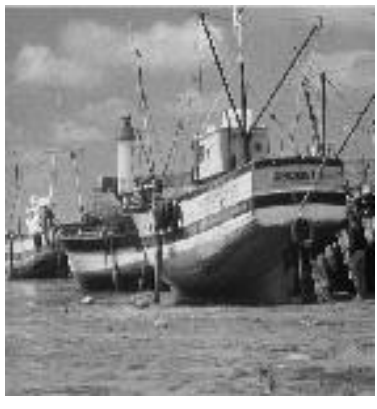

(d) Boat

Some robust geometrical works are used to illustrate the efficiency of the proposed system regarding robustness and imperceptibility, and the results have been compared. A fair comparison has been made using the case study with the related specification. MATLABR2014b is used for implementation. image to protect the integrity of watermarking techniques. If the are 262,144

(b) Contourlet transform has various levels, and different subbands are selected for watermarking.

(c) The partition size in the image is considered as $8 * 8$, and each partition is equally divided.

(d) Transparency and robustness are preserved using low transparency in Peak Signal to Noise Ratio (PSNR). wermarking techniques. The size of a gray-scale image is $32 * 32$. The image size selection satisfies the proposed system measured using different images with 256 gray levels including pictures such as Lena, cameraman, boat. Collection of these images is represented as the host image, and the watermarked image is obtained using the defined watermarking technique.

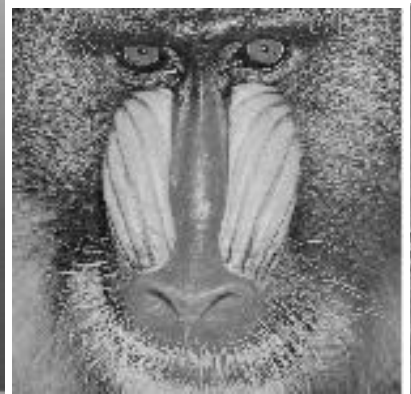

(c) Mandrill

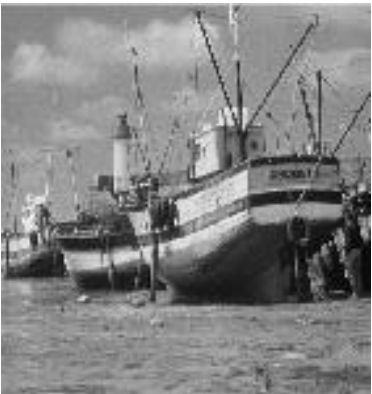

(d) Boat

Fig. 5: Watermarked images

Figure 4 and Figure 5 show the original and the watermarked images, and there is no visual difference between those two figures. Also, the PSNR is not so high. So, the transparency and the visual appearance is maintained for the watermarked image.
The imperceptibility of the watermarking algorithm is implemented where PSNR is described as

PSNR $=10 \log 10 \frac{\sum_{i=1}^{M} \sum_{j=1}^{N}(\mathrm{H}(\mathrm{i}, \mathrm{j}))^{2}}{\sum_{i=1}^{M} \sum_{j=1}^{N}\left(\mathrm{H}(\mathrm{i}, \mathrm{j})-H_{-} W(\mathrm{i}, \mathrm{j})\right)^{2}}$ 
When the PSNR value is higher, invisible watermarked image is obtained. PSNR value obtained by Fazli's scheme [20] is 48.46 whereas PSNR of proposed system is 56.41 .

Table 1: Proposed scheme PSNR vs. Fazli's scheme PSNR using an original image of size $512 * 512$

\begin{tabular}{|c|c|c|}
\hline Image & Fazli's scheme & Proposed scheme \\
\hline Cameraman & 48.46 & 56.41 \\
\hline Coco & 47.73 & 56.84 \\
\hline Mandrill & 49.45 & 56.06 \\
\hline Boat & 49.44 & 57.09 \\
\hline
\end{tabular}

Mean Square Error (MSE) of original and the watermarked image is calculated, where the coordinate pixel value of original and the watermarked model is given by $\mathrm{F}(\mathrm{i}, \mathrm{j})$ and $F^{W I}(\mathrm{i}, \mathrm{j})$ and the image size is $M^{*} N$.

$\operatorname{MSE}=\frac{1}{M * N} \sum_{i=0}^{M-1} \sum_{j=0}^{N-1}\left(\mathrm{~F}(\mathrm{i}, \mathrm{j})-F^{W I}(\mathrm{i}, \mathrm{j})\right)^{2}$

Table 2: Proposed scheme MSE vs. Fazli's scheme MSE using an original image of size $512 * 512$

\begin{tabular}{|c|c|}
\hline Image & Proposed scheme \\
\hline Cameraman & 0.8147 \\
\hline Coco & 0.9058 \\
\hline Mandrill & 0.1270 \\
\hline Boat & 0.9134 \\
\hline
\end{tabular}

The algorithm robustness is estimated for various attacks such as median filter, Gaussian filter, cropping and resizing. The improved results of PSNR are described in Table 1, and the improved results of MSE are described in Table 2. Embedded watermarks are extracted successfully in all manner, and it can be proved by calculating a correlation between embedded and extracted watermark for different attacks.

\section{Conclusion}

The existing scheme improves robustness against geometrical distortion. In host image, the watermark is embedded into four different segments to reduce cropping attack. The desired image is surrounded by the margin to resist geometric bouts like rotation, translation, and affine transformation. Combination of DCT, SVD, and DWT domains are implemented in the watermarking scheme to provide imperceptibility. In the proposed system, to improve visual quality, contourlet transform is used. After embedding the image or data, to enhance robustness contourlet transform is applied in the frequency domain. The optimal mapping function is obtained using contourlet transform which increases PSNR and hiding capacity with low distortion.

\section{References}

[1] D. Kundur and D. Hatzinakos, "Digital watermarking using multiresolution wavelet decomposition," Acoust. Speech Signal Process. 1998. Proc. 1998 IEEE Int. Conf., vol. 5, pp. 2969-2972, vol.5, 1998.

[2] S. Voloshynovskiy, F. Deguillaume, and T. Pun, "Content adaptive watermarking based on a stochastic multiresolution image modeling," Eur. Signal Process. Conf., vol. 2015-March, March, 2000

[3] P. Tao, A. M. Eskicioglu, and I. Science, "A robust multiple watermarking scheme in the Discrete Wavelet Transform domain," Opt. East 2004 Symp. Internet Multimed. Manag. Syst. V Conf. Philadelphia, PA, pp. 133-144, 2004.

[4] S. Pereira and T. Pun, "Fast Robust Template Matching for A ne Resistant Image Watermarks," pp. 1-12.

[5] V. Solachidis and I. Pitas, "Circularly symmetric watermark embedding in 2-D DFT domain,” IEEE Trans. Image Process., vol.
10, no. 11, pp. 1741-1753, 2001.

[6] S. Pereira, J. J. K. O. Ruanaidh, F. Deguillaume, G. Csurka, and T. Pun, "Template based recovery of Fourier-based watermarks using log-polarınand log-log maps," Proc. IEEE Int. Conf. Multimed. Comput. Syst., vol. 1, pp. 870-874, 1999.

[7] B. J. Falkowski and L.-S. Lim, "Image watermarking using the complex Hadamard transform," Proc. Int. Symp. Circuits Syst., vol. 4, p. IV--573-IV-576, 2000.

[8] S. Craver, N. Memon, B.-L. Yeo, and M. Yeung, "On the invertibility of invisible watermarking techniques," IEEE Int. Conf. Image Process., pp. 234-250, 1997.

[9] V. M. Potdar, H. Song, and C. Elizabeth, "A survey of digital image watermarking techniques," 2005 3rd IEEE Int. Conf. Ind Informatics, INDIN, vol. 2005, pp. 709-716, 2005.

[10] T.Padmapriya and V.Saminadan, "Utility based Vertical Handoff Decision Model for LTE-A networks", International Journal of Computer Science and Information Security, ISSN 1947-5500, vol.14, no.11, November 2016.

[11] A. Najih, S. A. R. Al-Haddad, A. R. Ramli, S. J. Hashim, and M. A. Nematollahi, "Digital image watermarking based on angle quantization in discrete contourlet transform," J. King Saud Univ. Comput. Inf. Sci., vol. 29, no. 3, pp. 288-294, 2017.

[12] L. Chen and J. Zhao, "Robust contourlet-based blind watermarking for depth-image-based rendering 3D images," Signal Process. Image Commun., vol. 54, August 2016, pp. 56-65, 2017.

[13] H. Sadreazami, M. O. Ahmad, and M. N. S. Swamy, "A study on image denoising in contourlet domain using the alpha-stable family of distributions," Signal Processing, vol. 128, pp. 459-473, 2016.

[14] Q. Zhang, W. Lu, and J. Weng, "Joint image splicing detection in DCT and Contourlet transform domain," J. Vis. Commun. Image Represent., vol. 40, Part B, pp. 449-458, 2016.

[15] H. Y. Patil, A. G. Kothari, and K. M. Bhurchandi, "Expression invariant face recognition using local binary patterns and contourlet transform," Optik (Stuttg)., vol. 127, no. 5, pp. 2670-2678, 2016.

[16] D. Li, L. Zhang, J. Yang, and W. Su, "Optik Research on waveletbased contourlet transform algorithm for adaptive optics image denoising," Opt. - Int. J. Light Electron Opt., vol. 127, no. 12, pp. 5029-5034, 2016.

[17] S. Biswas and J. Sil, "An efficient face recognition method using contourlet and curvelet transform," J. King Saud Univ. - Comput. Inf. Sci., 2017.

[18] D. Anandhi and S. Valli, "An algorithm for multi-sensor image fusion using maximum a posteriori and nonsubsampled contourlet transform R," Comput. Electr. Eng., vol. 65, pp. 139-152, 2018.

[19] X. Zhang, X. Zhang, M. Xu, H. Zhang, and X. Jiang, "Phase unwrapping in digital holograp0hy based on non-subsampled contourlet transform," Opt. Commun., vol. 407, September 2017 pp. 367-374, 2018

[20] S. Fazli and M. Moeini, "Optik A robust image watermarking method based on DWT, DCT and SVD using a new technique for correction of main geometric attacks," Opt. - Int. J. Light Electron Opt., vol. 127, no. 2, pp. 964-972, 2016.

[21] T. Padmapriya and V. Saminadan, "Improving Throughput for Downlink Multi user MIMO-LTE Advanced Networks using SINR approximation and Hierarchical CSI feedback", International Journal of Mobile Design Network and Innovation- Inderscience Publisher, ISSN : 1744-2850 vol. 6, no.1, pp. 14-23, May 2015.

[22] S.V.Manikanthan and T.Padmapriya "Recent Trends In M2m Communications In 4g Networks And Evolution Towards 5g", International Journal of Pure and Applied Mathematics, ISSN NO:1314-3395, Vol-115, Issue -8, Sep 2017. 